\title{
Educación social escolar en la construcción de cultura y educación para la paz: propuestas metodológicas de intervención socioeducativa
}

\author{
Social Education in the Construction of Culture and Education for Peace: \\ Methodological Proposals for Socio-Educational Intervention
}

Maria del Mar García-Vita

Universidad de Almería, España

http://orcid.org/0000-0002-1710-5592

Fanny Tania Añaños**

Universidad de Granada, España

http://orcid.org/0000-0001-7515-1987

Marta Medina García**

Universidad de Almería, España

https://orcid.org/000-0003-0520-3635

Artículo de investigación

Fecha de recepción: 29 de febrero de 2020

Fecha de aceptación: 7 de abril de 2020

* Doctora en Ciencias de la Educación por la Universidad de Granada. Profesora del Departamento de Educación de la Universidad de Almería. Miembro colaborador del Instituto de la Paz y los Conflictos (IPAz) de la Universidad de Granada. Su trabajo se desarrolla en el campo de la pedagogía y la educación social. Sus principales líneas de investigación son los estudios familiares, estudios de género, violencias, medio penitenciario, educación para la paz y prevención educativa. Correo electrónico: margvita@ual.es

** Doctora en Pedagogía por la Universidad de Granada. Profesora titular del Departamento de Pedagogía, subdirectora del Instituto de la Paz y los Conflictos (IPAz) y coordinadora del Máster Interuniversitario en Cultura de Paz, Conflictos, Educación y Derechos Humanos, de la Universidad de Granada (GI HISULA), Universidad Pedagógica y Tecnológica de Colombia. Desde la pedagogía y la educación social, trabaja en distintos campos de investigación e intervención socioeducativa. Actualmente dirige el Proyecto Nacional de Investigación sobre la Educación y Reinserción de Mujeres en Contextos Penitenciarios. Correo electrónico: fanntab@ugr.es

*** Doctora en Pedagogía por la Universidad de Jaén. Profesora del Departamento de Educación de la Universidad de Almería, Espańa. VI Premio cermi Derechos Humanos y Discapacidad por su tesis doctoral. Su principal línea de investigación es la inclusión educativa. Se ha desempeñado como coordinadora de proyectos educativos nacionales e internacionales en materia de educación. Correo electrónico: mmedina@ual.es 


\section{Para citar este artículo}

García-Vita, M., Añaños, F. T. y Medina García, M. (2020). Educación social escolar en la construcción de cultura y educación para la paz: propuestas metodológicas de intervención socioeducativa. Campos en Ciencias Sociales, 8(2), 47-71. DoI: https://doi.org/10.15332/25006681/6012

\section{RESUMEN}

La educación y cultura de paz se han instalado sólidamente en los contextos escolares a escala internacional y desde una multiplicidad de abordajes existentes: convivencia escolar, educación para la ciudadanía y derechos humanos, gestión o resolución de conflictos, estrategias participativas, entre otras. Ello es una muestra de la ampliación y complejización de los escenarios y actores educativos, así como de la realidad social, que constituye un terreno idóneo para el afianzamiento de propuestas desde la educación social. Dichas propuestas conciben la escuela como un espacio comunitario desde el cual se pueden construir apuestas educativas asentadas en la cultura de paz para la escuela y su entorno. El presente trabajo pretende hacer una revisión sobre el tema de la educación y cultura de paz en instituciones educativas, y cómo se pueden elaborar propuestas para su complementariedad, fortalecimiento y eficacia desde la educación social escolar.

Palabras clave: educación social, educación para la paz, cultura de paz, centros educativos, intervención socioeducativa.

\section{Abstract}

Education and culture of peace have been firmly established in school contexts at the international level and from a multiplicity of existing approaches: school coexistence, education for citizenship and human rights, conflict management or resolution, participatory 
strategies, among others. This is an example of the expansion and complexity of educational scenarios and actors, as well as of social reality, which constitute an ideal terrain for the consolidation of proposals from social education. These proposals conceive schools as a community space from which educational proposals can be built based on the culture of peace for the school and its environment. This paper aims to make a review of the subject of education and the culture of peace in educational institutions, and how proposals for their complementarity, strengthening, and effectiveness can be elaborated from the point of view of school social education.

Keywords: social education, peace education, culture of peace, educational centers, socioeducational intervention.

\section{BREVE RESEÑA METODOLÓGICA}

Se realizó un análisis sobre la educación y la cultura para la paz en instituciones educativas y sus métodos, con el fin de planificar y diseñar propuestas de acción para su complementariedad, fortalecimiento y eficacia desde la educación social escolar. Para ello se desarrolló una revisión especializada, cuyos resultados se estructuraron en varios subtemas, concatenados, que desembocaron en un apartado final, a modo de conclusión, sobre la indagación. Los criterios de búsqueda bibliográfica inicial incluyeron los constructos o palabras clave: "educación para la paz", "educación social" e "intervención socioeducativa".

El proceso de exploración se realizó en buscadores virtuales especializados y en bases de datos de distintas bibliotecas universitarias, seleccionando las publicaciones en español. Luego, se recopilaron 50 fuentes bibliográficas, teniendo como criterio de elección o inclusión su afinidad con el desarrollo del tema, ya que se ha dificultado perfilar un tema emergente como la educación social escolar y sus aportes a la educación para la paz. 


\section{EdUCACIÓN Y CULTURA DE PAZ EN CENTROS ESCOLARES}

La educación para la paz tiene su base epistemológica y metodológica en el marco de la pedagogía social, siendo uno de los campos profesionales y prácticos de la educación social y, a la vez, su objeto de estudio (Añaños-Bedriñana, 2012a; 2013). Así, la pedagogía social es entendida como la ciencia de la educación social que estudia las cuestiones inherentes a la socialización y la inadaptación. Está orientada a mejorar la calidad de vida de los individuos desde una perspectiva especial y fundamentalmente práctica (Pérez Serrano, 2005, p. 11), de modo que mantiene el objetivo de recuperar su dignidad e integrarlos socialmente. Por otro lado, la educación social procura la inserción del individuo en su medio, para que sea capaz de mejorarlo y transformarlo. Algunas de sus finalidades son: lograr la madurez social, promover las relaciones humanas y preparar al individuo para vivir en sociedad (Pérez Serrano, 2005, p. 11); todo ello amparado en los derechos humanos y el aprendizaje a lo largo de toda la vida (Ortega, 2005).

Las finalidades educativas generales y las propias de la educación para la paz confluyen, ya que ambas pretenden orientar el desarrollo de los individuos hacia la dignidad humana y los derechos humanos. Aquí la paz se entiende como garante de la transformación de actitudes, acciones, comportamientos y conductas (Cerdas, 2013). Los estudios de paz se han instalado en los contextos escolares a escala internacional y desde una multiplicidad de abordajes existentes: convivencia escolar, educación para la ciudadanía y derechos humanos, gestión o resolución de conflictos, mediación escolar, estrategias participativas, entre otras. Ello es una muestra de la ampliación y complejización de los escenarios y actores educativos. De esta manera emerge lo que Tuvilla (2004) ha denominado una sociedad educadora y la necesidad de educar para la complejidad (Morin, Ciruana y Motta, 2006), la cual no está exenta de conflictividad en tres dimensiones: con uno mismo y con los demás; con y desde las interacciones y estructuras sociales; y con el medio ambiente (Jares, 2001).

En este sentido, la educación debe promover acciones para generar actitudes críticas y de resistencia de la ciudadanía contra la violencia, los abusos, el odio y la dominación de unos sobre otros (Morin et al., 2006). La educación para la paz concentra su objetivo en esa transformación y cambio social desde el enfoque de la construcción 
de cultura de paz y respeto por la dignidad humana (Cerdas, 2013). Además, estudia y propone planteamientos, estrategias, métodos y acciones reales y coherentes, por lo tanto, basados en la evidencia y teniendo en cuenta las subjetividades-imaginarios, los contextos de las personas y su medio, en los que el conflicto es parte de la cotidianidad en toda sociedad y que hay que aprender a gestionarlo y transformarlo (Añaños-Bedrińana, 2012b).

La educación para la paz es un proyecto político y educativo que declara la lucha por la defensa de valores específicos como un medio para la construcción de una cultura de paz (Ospina, 2010; Unesco, 2011), la cual está asentada en las ideas de conflicto, así como la gestión y transformación de estos, de convivencia (Caballero, 2002), y promoviendo una validación constante de esos valores por parte de cada persona.

Los intentos de ejecutar acciones educativas integrales en pro de la cultura de paz requieren, desde la colectividad, reflexiones profundas sobre el sentido, finalidad y manera en que se articula el sistema educativo, pasando por la propia reconstrucción de la sociedad a partir de la cultura de paz y la práctica educativa (Hernández, Luna y Cadena, 2017). Esto representa varios retos, comenzando por concebir la escuela como un espacio comunitario desde el cual es posible irradiar propuestas educativas asentadas en la cultura de paz hacia su entorno. Es necesario considerar que dichas propuestas deben ser organizadas, coordinadas o dirigidas por y para la misma comunidad (Tuvilla, 2004). Lo escolar se torna en un microsistema dentro de un sistema social (nivel macro); y en ambos niveles es posible desarrollar la educación para la paz (Vidanes, 2007). Se destaca que esta solo tiene sentido si desemboca en la acción (Galtung, 1997) crítica, creativa, consensuada y no violenta de enfrentar los conflictos con uno mismo, con los demás y con la naturaleza (Rodríguez, 1995). La educación para la paz trasciende el ámbito escolar, se encuentra presente en los diversos procesos de socialización humana, y adquiere diversos significados en cada contexto (Ospina, 2010).

La participación es inherente a la educación para la paz y necesaria para el desarrollo de la capacidad crítica (Mayor, 2003). Es fundamental para la solidez del tejido comunitario, bajo los parámetros de pertenencia e implicación en los intereses, 
expectativas, problemas y necesidades comunes (Subirats, 2002); la escuela es una institución que puede articular y dinamizar acciones frente a ello, más allá de las aulas y lo curricular (Caballo y Gradaílle, 2008). Esto compromete a todas las personas de la comunidad educativa con la solución de los conflictos y problemas de los estudiantes, las familias y el resto de la comunidad (Medina y Cacheiro, 2010).

Según Cerdás (2003), podemos hablar de diferentes modelos de educación para la paz: modelo intimista, modelo conflictivo violento, modelo conflictivo no-violento y modelo sociocrítico. Para el objetivo de este trabajo, nos acogemos a este último modelo, que parte de las necesidades de los grupos humanos para una práctica educativa participativa, transformadora, emancipadora -en la línea del pensamiento freiriano- y que considera todo ámbito social y todo sujeto como posible escenario o agente para construir la paz (Cerdás, 2003; Ospina, 2010).

Con este trabajo queremos mostrar los aportes y respuestas que desde la educación social se dan a la necesidad de instaurar una perspectiva de paz en los centros educativos y ofrecer unas pautas generales para su puesta en marcha.

\section{EDUCACIÓN SOCIAL ESCOLAR: APORTES PARA LA EDUCACIÓN Y CULTURA DE PAZ}

Ramos (2003) pone de manifiesto que el sistema educativo se ha esforzado por disociar la dimensión afectiva y la cognitiva, priorizando la segunda y dejando como resultado una sociedad de escaso desarrollo emocional, de capacidad de autorregulación, resolución, consenso y negociación para afrontar los conflictos. De acuerdo con esto se forma lo que la misma autora ha denominado ciudadanos y ciudadanas analfabetos emocionales. Finalmente, sucede lo que la propia institución y el profesorado refiere: deficiencias a la hora de enfrentar los conflictos relacionales, lo cual está enraizado en un aislamiento del entorno próximo y alejamiento y dificultades de comunicación con el alumnado y sus familias (Menacho, 2013). 
La reconexión de los centros escolares con la sociedad debe ser un primer paso para la mejora de esta situación, y debe asumirse desde la bidireccionalidad de la relación y la doble intencionalidad, como lo define Ortega (1999, p. 99) "sociedad que eduque y dé una educación que socialice". Lo cual significa apostar por un ejercicio educativo integral, multidimensional, interdisciplinar, reflexivo, crítico y de acción (Caballo y Gradaille, 2008). Esto no difiere del propósito escolar, en el que confluyen la finalidad de la escolarización con la de la educación social -relativa a la socialización, el desarrollo de la personalidad o la identidad de los sujetos-, donde la distinción funcional y didáctica entre ambas es compleja (Ortega, 2005). La educación social en los centros educativos favorece en los individuos la adaptación a la vida social y sus normas, lo que debe permitirle su participación en la vida comunitaria y su capacidad de convivencia (Esteban, Amado y García, 2017).

La educación social es el fenómeno, la realidad, la praxis y la acción -con fundamentación pedagógica-, que se encuentra contextualizada y representa un factor de cambio social (Ortega 2005; Añaños-Bedriñana, 2012a, 2013). Como labor educativa, ha sido ejercida tradicionalmente con poblaciones en situación de riesgo y conflicto, y alejada del contexto escolar; no obstante, en los últimos años trabaja en y desde la escuela (Caride, Gradaille y Caballo, 2015), así como con cualquier grupo poblacional, no solo aquellos desfavorecidos o en riesgo social.

Al educador social se le define como un agente de cambio social, dinamizador de grupos sociales a través de estrategias educativas que ayudan a los sujetos a comprender su entorno social, político, económico y cultural, y a integrarse adecuadamente (Agencia Nacional de Evaluación de la Calidad y Acreditación [Aneca], 2005). Así pues, la intervención socioeducativa implica el reto de actuar en la sociedad, independientemente de las situaciones vitales en las que se encuentren los distintos colectivos, pues el objetivo principal es tener un futuro más digno y mejor para todos, garantizando la libertad individual y comunitaria desde los propios contextos socioculturales (Úcar, 2018).

Se considera que la educación social escolar no es antagónica a la escolar, ni trabaja aislada a ella; se deben crear puentes entre ambas y buscar la complementariedad de 
sus acciones. La educación social y la enseñanza son dos mundos paralelos dentro de un mismo universo (Galán, 2006) y están obligadas a entenderse. Se deben replantear las estructuras y esquemas institucionales y profesionalizadores tanto de docentes como de educadores sociales, entendiéndose desde la complementariedad (Parcerisa, 2008; Cuesta, Martínez, Cuesta, Sánchez y Orozco, 2017).

La educación social encuentra en los centros educativos un escenario privilegiado para desarrollar su labor, ya que constituye un derecho de la ciudadanía. Además, es generadora de contextos educativos y acciones mediadoras y formativas que posibilitan el desarrollo de la sociabilidad y la circulación social, así como la promoción cultural y social para ampliar las perspectivas educativas, laborales, de ocio y participación social (Foro Estatal Representativo de las Organizaciones de Educadores Sociales [Asedes] y Asociación Profesional de Educadores Sociales de Castilla la Mancha [Apescam], 2004).

La complejidad social trasciende las etiquetas y compartimentación de prácticas educativas; no obstante, la educación social y la escuela tienen que estar relacionadas -buscar la intersección entre ambas- pero manteniendo la distinción entre los dos campos (epistemológica, funcional y didácticamente). De esta manera, se incorporan saberes que pueden hacer más potente la integración social de todas las personas que forman parte de la comunidad (Merino, 2013; Ortega, 2005), pero sin confundirse con la vertiente social de una educación curricuralizada (Parcerisa, 2008).

La presencia de los educadores sociales surge como respuesta a las necesidades y problemáticas emergentes en la escuela (Terrón-Caro, Cárdenas-Rodríguez y Rodríguez-Casado, 2017). Dicha respuesta, en ocasiones, es incapaz por sí sola de gestionar las dificultades de convivencia, y encuentra limitaciones cuando pretende extender su acción fuera del entorno escolar (Parcerisa, 2008). La violencia escolar no es un fenómeno circunscrito exclusivamente a lo escolar, sino que es una realidad social. Por ello, es necesario analizar los factores de riesgo para la convivencia escolar y social a través de propuestas de acción socioeducativas para la prevención, que proporcionen herramientas para la gestión democrática, un uso responsable de los recursos y la construcción y adopción de un sistema de valores común (Merino, 2006; 
Ortega, 2005; Mayor, 2003). La perspectiva preventiva de la violencia es clave para el éxito de las acciones de cultura de paz que toman como referencia centros educativos, más aún si los profesionales de la educación consiguen la complicidad, trabajo en red y corresponsabilidad con los agentes familiares y comunitarios (Fernández-Herrería y López-López, 2014; Menacho, 2013).

Una idea pertinente es la de Fernández-Herrería y López-López (2014), quienes hablan del centro escolar como una unidad de cambio abierto a la comunidad, que aglutina dichos agentes y los implica en una estructura abierta al cambio, democrática, flexible y participativa, y con compromiso y presencia en el entorno próximo (Gairín y Armengol, 2008; San Fabián, 2011). Es posible enmarcar la acción socioeducativa en la educación a lo largo de toda la vida, como algo indispensable para la convivencia con la comunidad y la educación para la paz (Ramos, 2003). Los profesionales de la educación social son clave en la apertura del centro y la estructuración de propuestas comunitarias que apoyan la gestión de grupos y redes de acción (Parcerisa, 2003).

\section{CaRaCTERÍsTICAS Y PAUTAS PARA EL DISEÑO DE PROYECTOS SOCIOEDUCATIVOS}

La forma más idónea de sistematizar la intervención socioeducativa es un proyecto. Castillo y Cabrerizo (2011) lo definen como el conjunto integrado de procesos y actividades que pretende transformar un ámbito de la realidad social, atendiendo a carencias detectadas y proponiendo soluciones específicas a los problemas previamente diagnosticados a través de la intervención directa de agentes cualificados. El proyecto de tipo social debe definir los problemas sociales antes de iniciar el proyecto, sistematizar los objetivos de forma clara para que puedan ser evaluados en su consecución, identificar la población destinataria y sus características, establecer la temporalización y contar con los recursos necesarios (Cohen y Martínez, 2002).

Antes de ello, a la hora de planear el proyecto, debemos conocer las bases o fundamentos sobre los que se diseñan la mayor parte de las acciones socioeducativas. Estos sustentos son los encargados de dar forma y sentido a los procesos generales 
de cualquier intervención. Nos situamos en un enfoque crítico de intervención socioeducativa, el cual es considerado el más acorde con la naturaleza de la intervención y cuyo planteamiento básico entiende que un grupo o una colectividad tiende a la autodeterminación y a su mejora social, que cobra sentido al situarse en el contexto, en sus intereses y valores (Sáez, 1993). Este planteamiento se ve ampliado por Castillo y Cabrerizo (2011), quienes denominan la perspectiva interpretativa interaccionista, que busca comprender la realidad del grupo humano al que va dirigido y valorarla como un paso previo para su aplicación. También tiene en cuenta que cada colectivo posee unas actitudes, unos valores y una forma de acceder al conocimiento, y por lo tanto una forma diferenciada de reaccionar ante la intervención.

Luego, en la propia fase de diseño, se analizan los condicionantes de todo tipo que inciden en los procesos de intervención socioeducativa en contextos concretos y diferenciados. El diseńo debe abordarse desde una perspectiva sistémica, que apueste a resultados globales y sólidos, más allá de la propia acción educativa (PérezCampanero, 1991). El trabajo de diseño debe comenzar con una fase de trabajo prospectivo, en el que se fijan los motivos de la intervención (Rodríguez, 2009). Todo proyecto tiene un punto de partida que justifica la necesidad o urgencia de su implementación. Los motivos o razones que lo legitiman pueden ser variados (Castillo y Cabrerizo, 2011): satisfacer una necesidad relevante o combatir las barreras que impiden la satisfacción de esa necesidad, resolver una problemática dentro de un contexto y sistema concreto de valores, o introducir o promover un proceso de cambio que se considera necesario o deseable.

En términos generales, existen varias propuestas sobre los puntos tradicionales de la estructura de la intervención (Rodríguez, 2009; Castillo y Cabrerizo, 2011; Pérez Serrano, 2011); no obstante, nos inclinamos por la perspectiva de Añaños (2012b), a la que añadimos nuestros matices. Así, el proceso global de la acción-intervención socioeducativa comprende cuatro momentos:

1. Análisis de la realidad, también conocida como análisis de las necesidades o diagnóstico -sin embargo, es más empleada la primera-. Este momento responde a un principio paradigmático que afirma que antes de actuar es 
necesario conocer. Es decir, según Pantoja y Añaños (2010, p. 118), se actuará a partir de la realidad particular de las personas, respetando su situación y acomodándonos a sus perspectivas y limitaciones motivacionales.

2. Diseño y planificación de proyectos, programas o acciones, a partir de los resultados y conclusiones del análisis de la realidad, para responder positivamente a las cuestiones, problemas, necesidades o potenciales hallados. Su estructura contiene: título, resumen, justificación, objetivos, destinatarios, metodología, actividades, recursos (personales, materiales y financieros) e indicadores de evaluación.

3. Puesta en práctica-implementación de la acción diseñada.

4. Evaluación de la intervención, en todo su conjunto (del análisis de la realidad, del diseño, de la ejecución y de los logros obtenidos) o de cada etapa específica. De igual modo, puede ser una evaluación continua o de proceso, de pretest y postest, o de resultados. La evaluación puede ser realizada por el propio equipo, por entidades o personas externas, o equipos mixtos.

Existen algunas cuestiones por considerar a la hora de plantear una intervención socioeducativa. Así pues, debemos tener presente que, en la medida en que la sociedad va generando nuevas demandas de intervención cada vez más complejas y especializadas, las decisiones frente a cada uno de los elementos del proyecto serán aquellas que se adecúen a cada realidad social, como fundamento de cualquier intervención socioeducativa (Castillo y Cabrerizo, 2011), lo que hace que pueda ser modificada. Esto es menor o no se produce si se hace un buen análisis previo de la realidad.

Uno de los aspectos principales que debemos contemplar a la hora de diseñar e implementar un proyecto de intervención, es el papel que desempeñan los agentes de intervención, ya sean personales (profesional o equipo de profesionales) o institucionales (públicos o privados). Los agentes de intervención personales deben cumplir o ajustarse a un perfil concreto: a nivel personal, profesional, pedagógico, relacional y, por supuesto, actitudinal (Castillo y Cabrerizo, 2011). 
El paso a paso del diseño se inicia con la planificación y hace referencia a la elaboración de un plan que suponga un proceso de diseño, organización y preparación de una estrategia de acción, en el que se adopten las decisiones sobre la manera más conveniente de lograr los objetivos (Castillo y Cabrerizo, 2011). La intervención educativa es intencional, por lo que hay que definir, en primer término, qué es lo que pretendemos y cómo esperamos conseguirlo. Por lo tanto, la planificación se debe entender como un plan de acción que se irá adaptando al desarrollo práctico de este (Freixes y Aran, 2014). Esto supone una planificación abierta y flexible, que entienda y acoja la improvisación, puesto que en los procesos de intervención socioeducativa se producen distintas situaciones incontrolables que hacen que los cimientos básicos de la planificación puedan verse modificados (Forés, 2002).

Una parte relevante del proceso de planificación es el diagnóstico o evaluación inicial, que constituye una pieza clave en la adecuación de los proyectos de intervención socioeducativa a la práctica real. Este aspecto es especialmente problemático, ya que en muchas ocasiones se producen incoherencias o desconexión entre ese diagnóstico, el planteamiento de las intenciones y objetivos, y las estrategias previstas para llevarlo a cabo; cuando debe ser el que sustente la intervención y la provea de viabilidad y realismo (Freixes y Aran, 2014).

El éxito de una intervención depende, en gran medida, de la viabilidad de los objetivos establecidos. Es importante significar el qué, ya que los objetivos son el elemento de la planificación a partir del cual se articulan todos los demás necesarios, que se explicitan con la mayor claridad y precisión posibles. Para ser viable debe responder a las siguientes condiciones que señala Rodríguez (2009):

- Deben de ser alcanzables, viables y concretos. Por lo tanto, deben ser objetivos realistas que, además, serán objeto de evaluación específica.

- Deben reflejar de manera clara y expresa qué se quiere conseguir, pensando siempre en los destinatarios y no en los intereses de las personas responsables. 
- Deben estar contextualizados y ser coherentes en su propuesta con este aspecto de partida.

- Deben ser verificables; es decir, se debe comprobar fácilmente si se han logrado o no.

- Deben tener una redacción escueta y clara.

- Deben plantearse por nivel de prioridad. Por lo tanto, se debe distinguir entre objetivos generales o finales, y objetivos específicos, más precisos y concretos.

El cómo se va a desarrollar la intervención -la metodología empleada- constituye un elemento fundamental para la eficacia de la implementación del proyecto. La investigación-acción es la metodología más utilizada actualmente en la intervención socioeducativa, ya que en su planteamiento recoge los fundamentos más adecuados para el abordaje de esta cuestión; se lleva a cabo en y desde la práctica, y se basa en la reflexión.

Un planteamiento metodológico adecuado debe contar con las siguientes fases, las cuales son señaladas por Castillo y Cabrerizo (2011):

- Reflexionar sobre la filosofía y enfoque del proyecto, estrategias de intervención, modo de evaluación y recursos necesarios.

- Obtener información completa y exhaustiva de la población que se interviene.

- Tener en cuenta qué es necesario para alcanzar los objetivos y la gestión de recursos.

Por último, un componente muy importante y controvertido del plan de acción es la evaluación; esto se debe a la dificultad que conlleva en sí mismo este proceso. La evaluación afecta múltiples y variados factores que determinan los resultados 
(Gimeno y Pérez, 1985); de manera que para que esta se produzca, en términos de calidad, no debe ser un componente al margen de los demás, sino que tiene que estar presente a lo largo de todo el proceso y en íntima conexión con los demás elementos de un proyecto (Freixes y Aran, 2014). La evaluación, por lo tanto, se convierte en un componente relevante en la intervención socioeducativa, pues, como señala Rodríguez (2009), nos permite conocer el alcance de los logros de nuestra intervención, mediante un contraste entre lo que se pretendía y lo conseguido.

A continuación, señalamos los elementos que debe contemplar un diseño de evaluación, así como algunos de los instrumentos más utilizados:

- Elementos básicos del proceso de evaluación:

- Determinar los momentos de lo que se va a evaluar.

- Acordar los métodos de evaluación que se van a utilizar.

- Definir los elementos y aspectos que se van a evaluar.

- Instrumentos de evaluación:

- Registro de observación

- Entrevistas

- Discusión grupal

- Cuestionarios

- Anecdotario, etc.

En síntesis, las pautas para el diseńo de proyectos socioeducativos requieren fundamentalmente: elaborar un planteamiento global que recoja el conocimiento profundo de la situación de partida, establecer unos objetivos coherentes y contar con personas con competencias (personales y profesionales) suficientes para abordar la aparición de variables imprevistas que dificulten o modifiquen el planteamiento inicial. 


\section{INTERVENCIÓN SOCIOEDUCATIVA PARA LA CONSTRUCCIÓN DE UNA CULTURA DE PAZ EN CENTROS EDUCATIVOS}

La inserción de los educadores sociales en los centros educativos es una realidad en algunos territorios del Estado español; estos profesionales se incorporan a los equipos interdisciplinares de las escuelas (Terrón-Caro et al., 2017; López, 2013; Menacho, 2013). El objetivo de dicha integración es la adopción de acciones desde un enfoque preventivo y comunitario (Laorden, Prado y Royo, 2006), para la intervención en materia de convivencia, gestión de conflictos, educación en valores, factores de riesgo en menores, interculturalidad y diversidad, fracaso y absentismo escolar y dinamización, y participación familiar y comunitaria (Terrón-Caro et al., 2017; Junta de Andalucía, 2010). Además, como la naturaleza de la educación social preconiza, no se cierra ante posibles problemas, necesidades o realidades que puedan surgir. El educador social se incorpora de manera holística a la cotidianidad escolar. En este sentido, su labor no se circunscribe a acciones puntuales y aisladas que en materia de educación para la paz han resultado ser ineficaces o insuficientes, y que además deberían considerar e ir en consonancia con todo el marco normativo del centro y su modelo pedagógico-educativo (Caballero, 2002).

Tras la revisión, proponemos una serie de pautas que se deben tener en cuenta en el planteamiento, diseño y ejecución de proyectos de intervención socioeducativa en contextos escolares, para la construcción de una cultura de paz estructurada en los siguientes elementos.

\section{Una cultura de paz más allá de lo curricular: el educador social en los centros educativos}

Mucho se ha escrito sobre la necesidad de no limitar exclusivamente a las aulas el objetivo de construcción de paz en las instituciones educativas; es decir, que trascienda lo curricular (Caballero, 2002; Vidanes, 2007; Hernández et al., 2017). La dimensión cognitiva de la violencia y los conflictos no debe eclipsar la dimensión emocional (Ramos, 2003), ni el fomento de la participación, capacidad crítica, resolutiva y de gestión de situaciones de este tipo, desde la óptica de los derechos, la 
justicia social y ambiental, y las metodologías vivenciales y participativas (Hernández et al., 2017). Los niveles de intervención en materia de paz han sido definidos desde una perspectiva sistémica y clasificados en áreas por Ramos (2003): cuestiones sobre conflictos que emerjan; relaciones de las personas envueltas en conflictos; trabajo con el subsistema próximo, para que se asiente el cambio y no existan acciones contradictorias; y trabajo en el sistema, donde se analizan las injusticias estructurales sobre las que se asientan los conflictos. Todo ello se enmarca claramente en la acción socioeducativa y se excede de lo curricular.

Las funciones de los educadores sociales en centros educativos han sido descritas por varias normativas en el territorio español y también por varios autores, en un intento por dar forma a un campo relativamente novedoso. Nos quedamos con la amplia propuesta de Terrón-Caro y colaboradores (2017), de la que destacamos: la elaboración, el liderazgo y la evaluación de programas de convivencia y prevención de conflictos; la creación de espacios y de equipos de trabajo mediadores y negociadores para la eficacia en la gestión y transformación de conflictos; el diseño de estrategias para fomentar relaciones con el entorno social; la colaboración en acciones desarrolladas desde los departamentos de orientación o equipos directivos; el apoyo al profesorado en la generación de acciones curriculares y didácticas, así como proyectos de innovación docente, entre otras.

\section{Articulación de profesionales y entornos}

La construcción de cultura de paz desde lo educativo no solamente involucra a las instituciones formales, sino que compromete a todos los sectores de la sociedad (Hernández et al., 2017; Fernández-Herrería y López-López, 2014), siendo la educación social la vertiente educativa llamada a convertirse en el nexo en las relaciones escuela y comunidad. Esto con el fin de resituar la institución escolar en un proyecto integral de educación comunitaria (Caballo y Gradaílle, 2008).

Por un lado, abogamos por la implicación de roles profesionales con la incorporación de la figura del educador social y otros posibles pertenecientes a los servicios sociales, las organizaciones no gubernamentales, etcétera; y, por otro, por un llamado a la 
articulación con agentes educativos no profesionales, principalmente las familias (Hargreaves y Fink, 2006) y tejido asociativo del entorno, para asegurar el éxito de las propuestas.

Muy afín con nuestra perspectiva, Fernández-Herrería y López-López (2014) apuestan por el cambio de óptica en las relaciones con la comunidad educativa -que normalmente giran alrededor de la propia institución escolar y sus miembros, y con estructuras participativas mínimas y preestablecidas- y se comience a percibir una "nueva concepción del centro educativo, íntimamente vinculado con los contextos (familiares, comunitarios y ecológicos) con relaciones sociales más fluidas, complejas, cercanas a lo personal, multidireccionales y menos jerarquizadas, en una comunidad educativa ampliada” (pp. 134-135).

\section{Objetivos asentados en un diagnóstico socioeducativo contextualizado}

Decíamos que el éxito de una intervención depende, en gran medida, de los objetivos establecidos, los cuales se deben enfocar en los intereses de los beneficiarios, estar contextualizados tras una fase de diagnóstico y ser verificables, viables y organizados según la prioridad (Rodríguez, 2009). Los objetivos en una propuesta socioeducativa encaminada a la cultura de paz deben orientarse a favorecer la emancipación de la persona y de la humanidad en su conjunto (Vidanes, 2007).

\section{Los contenidos: relacionales y transversales}

En el apartado Articulación de profesionales y entornos mencionamos que las funciones de los educadores sociales son variadas. Entre ellas están: apoyar al profesorado a introducir y transversalizar contenidos propios de la paz en el currículo escolar, y liderar proyectos propiamente socioeducativos (con objetivos de tipo social y relacional).

Como punto de partida, el Manifiesto 2000 de la Unesco, Por una cultura de paz y no violencia, considera que una propuesta de este tipo debe incidir en el respeto de la vida y dignidad; rechazar la violencia; cultivar la generosidad; evitar la exclusión, 
la injusticia y la opresión; defender la libertad de expresión y la diversidad cultural; promover el consumo responsable; y contribuir al desarrollo de la comunidad. La educación para la paz armoniza lo personal (educación moral, sexual y de la salud), lo social (educación vial, del consumidor e intercultural) y lo ambiental (educación ambiental) (Vidanes, 2007). Todas estas áreas de acción son terreno tradicional del trabajo de la educación social, sobre todo desde la perspectiva preventiva. Pero, además de manera más concreta, la acción socioeducativa para la prevención y el tratamiento de conflictos violentos se puede trabajar a través recursos como la educación en valores sociales, la utilización del conflicto como recurso de aprendizaje, y la mediación y diseño de programas de convivencia (Merino, 2006).

A través de la labor educativa se deben desarrollar habilidades para la búsqueda de soluciones creativas, la negociación, el diálogo, la mediación en relación con los conflictos, la violencia, la explotación, el daño ambiental y oponerse a todo lo que sea contrario a la vida y a la dignidad humana. Esto con el fin de favorecer la transición hacia una cultura de paz y considerar que las necesidades de los demás toman un papel tan relevante como el de las necesidades propias (Caballero, 2002; Mayor, 2003).

\section{Metodologías activas y participativas}

Concebir el centro escolar como una unidad de cambio requiere una organización institucional flexible, democrática, abierta, autónoma, comprometida y con presencia y liderazgo educativo y social (Fernández-Herrería y López-López, 2014; Gairín y Armengol, 2008; San Fabián, 2011).

La intervención socioeducativa comprende la realidad desde la perspectiva ecológicasistémica, lo cual implica entender la violencia como un problema complejo, no circunscrito al ámbito escolar y que requiere de estrategias individuales de orientación dirigidas a estudiantes; estrategias relacionales, como la mediación escolar o la mediación entre iguales, para incidir en las relaciones de víctimas y agresores; estrategias comunitarias para concienciar y proponer acciones de rechazo a la violencia y apoyo a víctimas; y estrategias de sensibilización de la sociedad como, por ejemplo, campañas (Tuvilla, 2004, p. 20). 
Las opciones metodológicas disponibles para el planteamiento de un proyecto socioeducativo no son definidas a priori, ya que deben de ser un elemento secundario y no por ello menos importante, que sea pertinente para la población, objetivos y diagnóstico elaborados.

\section{La evaluación socioeducativa}

El último elemento que se debe tener en cuenta es la evaluación, la cual se perfila como un proceso que puede albergar varios momentos, métodos e instrumentos, de acuerdo con los aspectos a evaluar (Rodríguez, 2009). Principalmente, este proceso tiende a lo cualitativo, entendiéndose, como una evaluación continua, con el objetivo -entre otros- de mejorar y reorientar, si fuese necesario, durante la propia ejecución del proyecto, a modo de autoevaluación en la que se involucra a los participantes, valorando los puntos fuertes y débiles, y anticipando los efectos y consecuencias del proyecto (Pascual, 2007). En consonancia con los objetivos propios de este trabajo, se debe orientar esa evaluación según el tipo de acción desarrollada y los objetivos trazados para la construcción de paz en las instituciones escolares.

Con todo, concluimos que los principios y acciones para lograr los procesos de convivencia social en cultura de paz se deben plantear en el marco de un trabajo conjunto de los entes responsables, la sociedad global y cada una de las personas, teniendo en cuenta la cultura, la historia, la economía, la política, las representaciones sociales, el género, etc. En estas perspectivas, la potenciación o mejor aprovechamiento y coordinación de los recursos, las oportunidades y las intervenciones sociales corresponden a unos criterios de calidad y adecuación concreta a la persona y a las medidas.

Asimismo, es importante favorecer un desarrollo integral de las personas, que considere sus habilidades, capacidades, intereses, motivaciones y competencias, tanto personales como sociales, en un proceso continuo y liberador. Para ello, la pedagogía social y la educación social, en su dimensión teórica y práctica, no solo necesita desarrollar fundamentos, métodos, técnicas, etc., sino un pensamiento pedagógico con dimensiones críticas hacia las situaciones sociales que provocan el proceso de violencia, marginación y exclusión en sus diversas manifestaciones, grados y momentos (Añaños, 2012b). 


\section{REFLEXIONES FINALES}

La revisión llevada a cabo presenta limitaciones ya que, como decíamos al inicio del artículo, la educación social escolar -a pesar de ser un campo de acción manifiesto en la actualidad-carece de una definición unánime a nivel teórico y profesional, así como de claridad sobre los temas y funciones por desarrollar en los contextos escolares. Es por ello que apostar por realizar esta revisión teórica sobre las respuestas que se dan al desarrollo de la educación para la paz desde la educación social escolar es, en sí mismo, un intento por afianzar la práctica socioeducativa en centros escolares desde un enfoque de cultura de paz. Los bajos niveles de concreción en el tema y la escasez de fuentes que realmente trabajan, han hecho que la revisión bibliográfica haya sido reorientada a lo largo de su desarrollo, contando con reglas mínimas de inclusión y exclusión, y con un amplio nivel de apertura a las aristas del tema planteado. No obstante, los resultados de este trabajo permiten reconocer las potencialidades del contexto escolar en la acción socioeducativa para una cultura de paz, como un paso clave en su definición y fomento.

Vivimos en una sociedad con luces y con sombras, donde el conflicto está presente. No obstante, este fortalece el análisis, a partir del cual se puede replantear dicha realidad, su concienciación y el cambio. Todo ello con el fin de que el ser humano sea capaz de transitar críticamente y transformar su realidad, así como promocionar, participar y dinamizar su calidad de vida.

\section{REFERENCIAS}

Agencia Nacional de Evaluación de la Calidad y Acreditación (Aneca). (2005). Libro Blanco Titulo de Grado en Pedagogía y Educación Social. Madrid: Aneca.

Añaños-Bedriñana, F. (2012a). Pensamiento y acción socioeducativa en Europa y España. Evolución de la Pedagogía y Educación Social. Revista de Historia de la Educación Latinoamericana, 14(18), 119-138. 
Ańańos-Bedriñana, F. (2012b). Violencias y exclusiones. Enfoque socio-educativo y de la paz. Convergencia. Revista de Ciencias Sociales, 19(59), 13-41. Recuperado de http://www.scielo.org.mx/scielo.php?pid=S1405-14352012000200001\&script=sci_abstract

Añaños-Bedrińana, F. (2013). ¿Qué es la Pedagogía y Educación Social? Aproximaciones introductorias y conceptuales. En D. Soto Arango y A. Martínez Moya (eds.), La construcción de la nación iberoamericana. Siglos XIX-XX. Comparaciones y conexiones. Colección de Cuadernos de Vendimia, tomo III (pp. 91-112). Tunja: RUDECOLOMBIA.

Caballero, A. (2002). Educación para la paz y la convivencia: implicaciones para la gestión de conflictos escolares. Cultura y Educación, 14(4), 403-411.

Caballo, B. y Gradaille, R. (2008). La Educación Social como práctica mediadora en las relaciones escuela-comunidad local. Pedagogía Social. Revista Interuniversitaria, 15, 45-55.

Caride, J. A., Gradaille, R. y Caballo, B. (2015). De la pedagogía social como a la educación social como Pedagogía. Perfiles Educativos, XXXVII(148), 4-11.

Castillo, S. y Cabrerizo, J. (2011). Evaluación de la intervención socioeducativa: agentes, ámbitos y proyectos. Madrid: Pearson.

Cerdas, E. (2013). Educación para la paz: fundamentos teóricos, epistemológicos y axiológicos. Revista Latinoamericana de Derechos Humanos, 24(1-2), 189-201.

Cohen, E. y Martínez, R. (2011). Manual de formulación, evaluación y monitoreo de proyectos sociales. Cepal.

Cuesta, M. C., Martínez, M. A., Cuesta, J. L., Sánchez, S. y Orozco, M. L. (2017). El educador social en la enseñanza secundaria. La mediación escolar como alternativa a la resolución de conflictos. EHQUIDAD. Revista Internacional de Politicas de Bienestar y Trabajo Social, (7), 145-174.

Esteban, M., Amado, L. V. y García, R. (2017). Mediación en centros escolares. El papel de la Educación Social. Cuestiones Pedagógicas, 26, 83-96. 
Fernández-Herrería, A. y López-López, M. C. (2014). Educar para la paz. Necesidad de un cambio epistemológico. Convergencia. Revista de Ciencias Sociales, 64(21), 117-142.

Forés, A. (2002). Quan la didàctica porta el nomd'educació social. Barcelona: Fundación Pere Tarrés.

Freixes, N. G. y Aran, A. P. (2014). La intervención socioeducativa desde una mirada didáctica. Edetania: estudios y propuestas socio-educativas, (45), 55-72.

Gairín, J. y Armengol, C. (2008). Estrategias de formación para el cambio organizacional. Madrid: Walters Kluwer.

Galán, D. (2006). Modalidades de participación do/a educador/a social na escola. En M. Castro, X. M. Malheiro, y X. Rodríguez (coords.), A escola, ¿punto de encontro entre o profesorado e educado-res/as sociais? (pp. 48-52). Santiago de Compostela: Nova Es-cola Galega - Cesga.

Galtung, J. (1997). La educación para la paz sólo tiene sentido se desemboca en la acción. El correo de la Unesco.

Gimeno, J. y Pérez, A. (coords.). (1985). La enseñanza: su teoria y su práctica. Madrid: Akal.

Hargreaves, A. y Fink, D. (2006). Sustaining leadership. Phi Delta Kappan, 84(9), 693-700.

Hernández, I., Luna, J. A. y Cadena, M. C. (2017). Cultura de paz: Una construcción desde la educación. Revista Historia de la Educación Latinoamericana, 18(28), 149-172.

Jares, X. (2001). La educación para la paz en el umbral del nuevo siglo: retos y necesidades. En Centro Pignatelli (ed.), La Paz es una Cultura: Seminario de Investigación para la Paz (pp. 507-536). España: Octavio y Félez, S. A.

Junta de Andalucía. (2010). Instrucciones de 17 de septiembre de 2010 de la Dirección General de Participación e Innovación Educativa por la que se regula la intervención del educador y educadora social en el ámbito educativa. Andalucía: Junta de Andalucía 
Laorden, C., Prado, C. y Royo, P. (2006). Hacia una educación inclusiva. El papel del educador social en los centros escolares. Pulso, 29, 77-93.

López, R. (2013). Las educadoras y los educadores sociales en centros escolares en el estado español. RES. Revista de Educación Social, 16.

Mayor, F. (2003). Educación para la paz. Revista Educación XXI, 6, 17-24.

Medina, A. y Cacheiro, M. L. (2010). La prevención de la violencia: la implicación de la comunidad educativa para evitar situaciones de acoso escolar. Bordón, 61(1), 93-107.

Menacho, S. (2013). El educador social y la escuela. Justificación de la necesidad de la Educación Social en la escuela. Revista de Educación Social, 16.

Merino, J. V. (2006). La violencia escolar: análisis y propuestas de intervención socio-educativa. Santiago de Chile: Arrayán.

Merino, R. (2013). La educación social en la escuela / la escuela en la educación social. Revista de Educación Social, 16, 1-9.

Morin, E., Ciurana, E. y Motta, R. (2006). Educar en la era planetaria (1. reimpresión). Barcelona: Editorial Gedisa, S. A.

Ortega, J. (2005). La educación a lo largo de la vida: la educación social, la educación escolar, la educación continua... todas son educaciones formales. Revista de Educación, 338, 167-175. Recuperado de https://pdfs.semanticscholar.org/a8e7/695d10ae9097d06be36 6d1abf0628b5d6f6d.pdf

Ospina, J. (2010). La educación para la paz como propuesta ético-política de emancipación democrática. Origen, fundamentos y contenidos. Universitas. Revista de Filosofía, Derecho y Politica, 11, 93-125. 
Pantoja Vargas, L. y Añaños-Bedriñana, F. T. (2010). Actuaciones socioeducativas con menores vulnerables, en riesgo, relacionados con las drogas. Reflexiones críticas. Pedagogía Social. Revista Interuniversitaria, 17, 109-122.

Parcerisa, A. (2008). Educación Social en y con la institución escolar. Pedagogía Social. Revista Interuniversitaria, 15, 15-27.

Pascual, B. (2007). La evaluación de la intervención comunitaria: un marco para la reflexión. Pedagogía Social. Revista Interuniversitaria, 14, 129-138.

Pérez Serrano, G. (2005). Presentación. Revista de Educación, 338, 7-18. Recuperado de http://www.educacionyfp.gob.es/dam/jcr:9488fcdd-a368-46fb-ae04-cd0deaa16860/ re33601-pdf.pdf

Pérez Serrano, G. (2011). Diseño e implementación de proyectos de intervención. En G. Pérez Serrano (coord.), Intervención sociocomunitaria (pp. 693-714). Madrid: Uned.

Pérez-Campanero, M. P. (1991). Cómo detectar las necesidades socioeducativas de intervención socioeducativas. Madrid: Narcea.

Ramos, E. (2003). El desarrollo de la educación por la paz: un camino de obstáculos y oportunidades. Revista Educación XX1, 6, 129-146.

Rodríguez, J. M. (2009). Guía para el diseño de programas socioeducativos de atención a la infancia. Foro de educación, 7(11), 287-301.

Rodríguez, M. (1995). La educación para la paz y el interculturalimo como tema transversal. Barcelona: Oikos-Tau, S.L.

Sáez Carreras, J. (1993). La intervención socioeducativa: entre el mito y la realidad. Pedagogía social: revista interuniversitaria, 8, 89-106.

San Fabián, J. L. (2011). El papel de la organización escolar en el cambio educativo: la inercia de lo establecido. Revista de Educación, 356, 41-60. 
Subirats, J. (2002). Gobierno local y educación. La importancia del territorio y la comunidad en el papel de la escuela. En Humet (coord.), Educación y territorio. El factor proximidad $y$ de comunidades en las politicas educativas (pp. 23-41). Barcelona: Ariel.

Terrón-Caro, T., Cárdenas-Rodríguez, R. y Rodríguez-Casada, R. (2017). Educación intercultural inclusiva. Funciones de los/as educadores/as sociales. Pedagogía social: revista interuniversitaria, 29, 25-40.

Tuvilla, J. (2004). Guía para elaborar un Proyecto Integral de Escuela: Espacio de Paz. Andalucía: Consejería de Educación y Ciencia de la Junta de Andalucía.

Úcar, X. (2018). Metáforas de la intervención socioeducativa: implicaciones pedagógicas para la práctica. Revista española de pedagogía, 76(270), 209-224.

Unesco. (1999). Manifiesto 2000 para una cultura de paz y no violencia. Recuperado de http:// manifesto2000.unesco.org/sp/sp_manif2000_t.htm

Unesco. (2011). IV Jornadas de Cooperación Iberoamericana educación para la paz, la convivencia democrática y los derechos humanos. Montevideo: Oreal/Unesco.

Vidanes, J. (2007). La educación para la paz y la no violencia. Revista Iberoamericana de Educación, 42(2). 